\title{
On the Goal Domain in the RM-ODP Enterprise Language: An Initial Appraisal based on a Foundational Ontology
}

\author{
João Paulo A. Almeida, Evellin Cristine Souza Cardoso, Giancarlo Guizzardi \\ Ontology and Conceptual Modelling Research Group (NEMO) \\ Computer Science Department, Federal University of Espírito Santo (UFES) \\ Av. Fernando Ferrari, s/n, Vitória, ES, Brazil \\ jpalmeida@ieee.org, ecardoso@inf.ufes.br, gguizzardi@acm.org
}

\begin{abstract}
The "goal domain" addresses objectives in a broad scope ranging from high-level statements expressing the vision and mission of an organization (or community) to declarations of the results that must be achieved by business process execution. This paper reviews the support for the goal domain in ISO RM-ODP. We analyze the conceptualization for goals (objectives) in the reference model's Enterprise Viewpoint using a fragment of the Unified Foundational Ontology (UFO) that deals with some aspects of social reality and intentionality. The interpretation of the RM-ODP concepts in terms of this foundation may help to provide a sound underpinning for these concepts and support the identification of possible extensions of the reference model.
\end{abstract}

Keywords: objectives, goals, enterprise viewpoint, foundational ontology, social concepts

\section{INTRODUCTION}

Among the various architectural domains, the domain of "motivation" has been recognized as an important element of enterprise architectures [41], having been addressed to different extents in Zachman framework's "why column" [37], ARIS framework's "objectives diagram" [32], DoDAF and MODAF's "strategic views" [7, 38], OMG's Business Motivation Model (BMM) [29], ArchiMate's "ARMOR extensions" [30] and, finally, RM-ODP's "enterprise viewpoint" [24].

Goal modelling explicitly captures the goals of an enterprise, documenting an enterprise's strategy [3] and helping in clarifying interests and intentions from different stakeholders [22]. The definition of goals is related with objectives in a broad scope within the enterprise, i.e., goal statements range from high-level concerns in an enterprise (expressing the vision and mission of the organization) to declarations of the values that must be achieved by business process execution on behalf of stakeholders.

Goals are intrinsically related to other elements of the enterprise: while the goal domain focuses on "why" [22, 40], a behavioural domain focuses on "how" the enterprise organizes work and resources to fulfil its strategies [35] and a structural domain focuses on "who" is responsible to perform organizational activities that ultimately realize the enterprise's goals.

By aligning goals with other elements in enterprise architectures such as physical or informational resources, one can visualize how the elements of the enterprise are manipulated in business processes in order to attain enterprise's goals. In other words, goal modelling is motivated by the necessity of providing a strategic dimension for the enterprise's elements, acknowledging the fundamental role of the whole set of elements of an enterprise architecture in achieving the enterprise's goals.

As a comprehensive framework for open system specification, the RM-ODP addresses this strategic dimension by including a concept of "Objective" in its enterprise language. Further, the reference model acknowledges the importance of this concept by relating it to key elements of the enterprise viewpoint, including community - and thus the enterprise objects in the community including "the ODP system" - policy, role and process definition. In a nutshell, communities are defined to achieve certain objectives. These objectives influence the definition of the policies and roles in the community, which affect the behaviour of the enterprise objects to favour the satisfaction of community objectives.

This paper reviews the support for the objective concept in the enterprise viewpoint in order to consider how the goal domain is addressed in RM-ODP. We analyze the conceptualization for goals (objectives) in the reference model's Enterprise Viewpoint using the Unified Foundational Ontology (UFO) [16, 17] as our semantic foundation. UFO is a philosophically and cognitively wellfounded reference ontology that incorporates concepts to deal with some aspects of social reality and intentionality [17], including the notion of goal.

We argue that interpretation of the RM-ODP concepts in terms of this foundation may help to provide a sound underpinning for these concepts and strengthen RM-ODP as a basis for communication and consensus. Our interpretation is in line with [27], which points out "that enterprise behaviour has an additional dimension of social behaviour to express how enterprise objects can strive towards objectives, choose to undertake obligations, and make plans correspondingly." Further, the analysis performed here supports us in the identification of topics for further investigation regarding possible extensions of RM-ODP.

This paper is further structured as follows: Section II reviews how goal-related concepts are addressed in the enterprise viewpoint. Section III presents the semantic foundation to be employed in our analysis. Section IV presents the analysis of the reference model's goal-related concepts. Finally, Section V presents our conclusions and outlines some topics for further investigation. 


\section{GOAL CONCEPTS IN THE REFERENCE MODEL}

The reference model defines a framework for the specification of ODP systems which has five viewpoints, called enterprise, information, computational, engineering and technology. In each of the aforementioned viewpoints, a viewpoint language defines the concepts and rules for developing, representing and reasoning about the specification of an ODP system from that viewpoint [24].

This section reviews the enterprise viewpoint as defined in ITU-T Rec. X.911 / ISO/IEC 15414 [24] with an emphasis on the concepts used to address strategic concerns.

\section{A. ISO RM-ODP Enterprise Language}

The enterprise viewpoint includes a concept of Objective, which is defined as "practical advantage or intended effect, expressed as preferences about future states."

Objectives in an enterprise specification are closely associated with the notion of community which is a fundamental structuring concept for enterprise specifications: "a community is a configuration of enterprise objects that describes a collection of entities (e.g. human beings, information processing systems, resources of various kinds and collections of these) that is formed to meet an objective."

An enterprise specification states the objective of a community, how it is structured, what it does, and what objects comprise it (including an enterprise object which is the ODP system). Every community in an enterprise specification has exactly one objective. The purpose of an ODP system is expressed as one or more objectives (or subobjectives) of the community or set of communities in which the ODP system fulfils roles.
The objective of a community is expressed in a contract which specifies how the objective can be met. More specifically, policies in the enterprise contract constrain the behaviour and membership of communities in order to make them achieve their objectives. The behaviour that the community is expected to exhibit is defined in terms of roles or processes or both, policies, and the relationships of these.

An enterprise specification may decompose the objective of a community into sub-objectives (See Figure 1 for the refinement relation between Objectives).

A sub-objective may be assigned to a collection of roles; in that case, the behaviour of the collection of roles is specified to meet the sub-objective and the sub-objective is met by the collection of objects performing the actions of the collection of roles (see association between Objectives and Behaviours identified by Roles in Figure 1).

Further, a sub-objective may be assigned to a process ("A collection of steps taking place in a prescribed manner and leading to an objective."). In that case, the process is specified to meet the sub-objective and the sub-objective is met by the actions of objects performing the process. In this case, the sub-objective defines the state in which the process terminates.

In a hierarchical perspective on an enterprise specification, a community may be regarded as a composite community-object which may fulfil a role in another community. In this case, the objective of the community of which the community-object is an abstraction is consistent with any sub-objectives assigned to that role in the other community.

Figure 1 presents a (non-normative) UML diagram

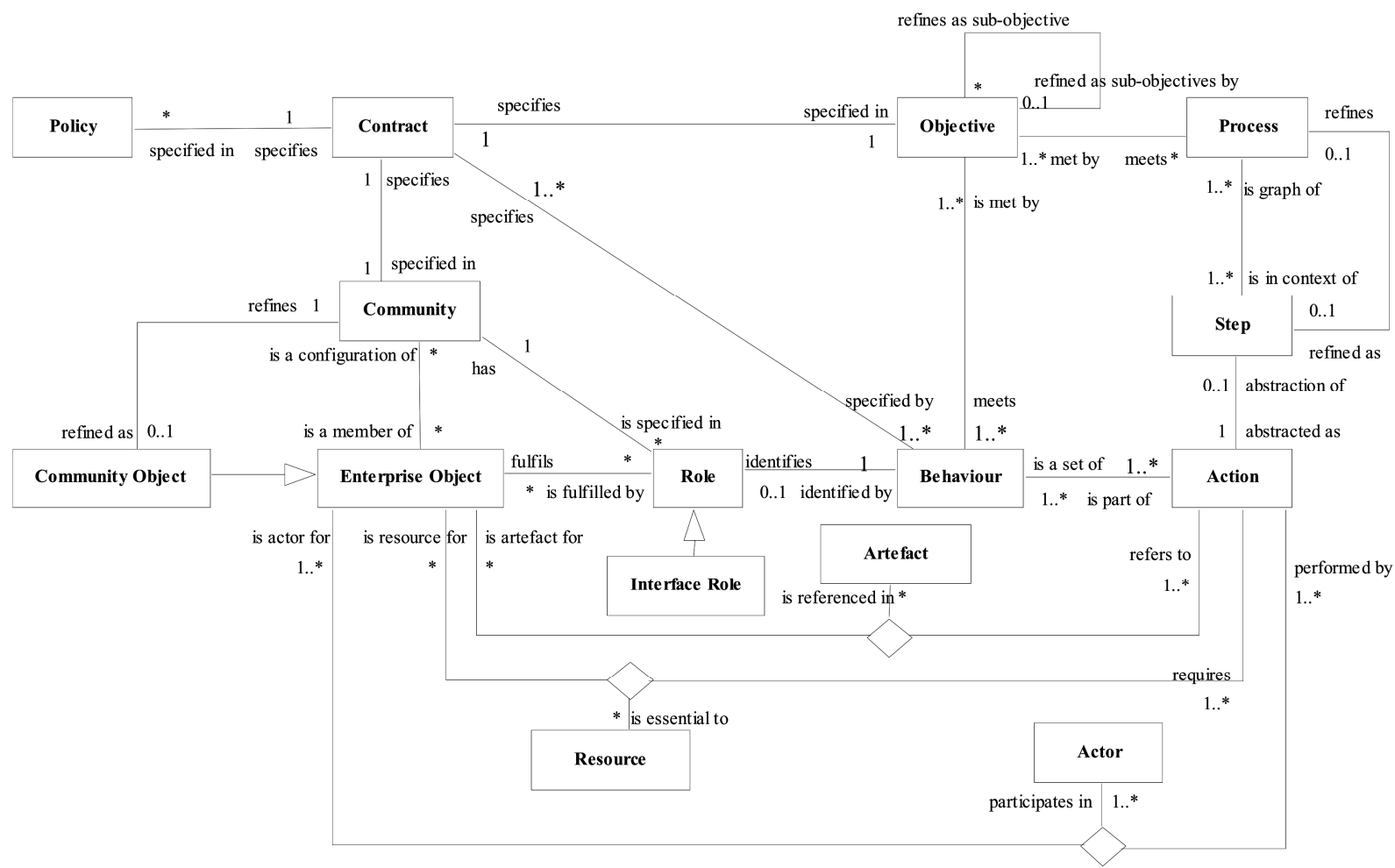

Figure 1 - RM-ODP Community and Behaviour Concepts [24] 
included in the standard to represent relationships between the main concepts used in modelling the behaviour of a community. (The labels at association ends closer to a class indicate the verb phrase that should be applied to the noun that names a class. Examples are: "Process" "meets" "Objective"; "Role" "identifies" "Behaviour", "Objective" "is met by" "Behaviour" and "Contract" "specifies" "Objective".)

\section{ONTOLOGICAL FOUNDATIONS}

Ontology is that branch of philosophy that "studies the most pervasive features of reality" [13]. The idea behind ontological analysis is to provide a sound foundation for modelling concepts, if assumed that such concepts are aimed at representing reality [13].

Several recent efforts have shown the benefits of ontological analysis [2, 14, 15, 19, 31, 34], which include: (i) the rigorous definition of modelling languages in terms of real-world semantics for their modelling constructs; (ii) the identification of inappropriate constructs which can reveal problems in the definition, interpretation and/or usage of concepts, and (iii) recommendations for language improvement to reduce lack of expressivity, ambiguity and vagueness.

Besides providing a clear semantic account for language constructs, ontological analysis has shown to be useful for language comparison and integration $[10,13,16,21]$ and, when the ontology itself is described in a formal language, as a basis for model simulation and validation [5].

Before we perform an analysis of the enterprise language concepts, we present here an ontological foundation on which we basis our analysis. We will use concepts of a philosophically and cognitively well-founded reference ontology called Unified Foundational Ontology (UFO) discussed in depth in $[16,17,20]$. UFO unifies several foundational ontologies and has been developed with the purpose of providing foundations for conceptual modelling based on philosophically well-founded principles as well as capturing the ontological distinctions underlying human cognition and common sense. Further, its choice has also been justified by its successful employment in previous works to evaluate, re-design and integrate the models of conceptual modelling $[16,17]$ and enterprise modelling [2, 31]. The UFO fragment presented in this section (and depicted in Figures 2 and 3) deals with some aspects of social reality and intentionality. For a fuller discussion regarding this foundational ontology, one should refer to the aforementioned references.

\section{A. Basic Elements}

We start with the fundamental distinction between universals and individuals. The notion of universal underlies the most basic and widespread constructs in conceptual modelling. Universals are predicative terms that can possibly be applied to a multitude of individuals, capturing the general aspects of such individuals. Individuals are entities that exist instantiating a number of universals and possessing a unique identity. Individuals can be further classified into Endurants and Events (also known as Perdurants).
Endurants are individuals said to be wholly present whenever they are present. Endurants are in time in the sense that if we say that in circumstance $\mathrm{cl}$ an endurant e has a property $\mathrm{P} 1$ and in circumstance $\mathrm{c} 2$ the property $\mathrm{P} 2$ (possibly incompatible with $\mathrm{P} 1$ ), it is the very same endurant $\mathrm{e}$ that we refer to in each of these situations. Examples of endurants are a house, a person, the moon, an enterprise and an ODP system.

A Substantial is an Endurant that does not depend existentially on other Substantials ${ }^{1}$, roughly corresponding to what is referred by the common sense term "object". In contrast with Substantials, we have Moments which are existentially dependent entities. For a Moment $\mathrm{x}$ to exist, another individual must exist, named is bearer. Examples of Substantials include a person, a house, a planet, and the Rolling Stones; examples of Moments include John's weight and John and Mary's marriage.

Moments can be classified into Intrinsic Moments, when existentially dependent on a single entity and Relational Moments (or Relators) otherwise. John and Mary's marriage is an example of a Relator that is dependent on both John and Mary. In certain situations, when bearing Relational Moments, substantial individuals are said to instantiate a Role Universal. So, in the aforementioned example, John is not only an instance of a "Person" universal but also an instance of a "Husband" universal, while Mary is an instance of a "Wife" universal.

Situations are special types of endurants. These are complex entities that are constituted by possibly many endurants (including other situations). Situations are taken here to be synonymous to what is named state of affairs in the literature, i.e., a portion of reality that can be comprehended as a whole. Examples of situations include "John being with fever and influenza", "John being in the same location as Paul while Mary is in the same location as David", "Mary being married to Paul who works for the University of Twente".

Events (Perdurants), in contrast with Endurants, are individuals composed by temporal parts, they happen in time in the sense that they extend in time accumulating temporal parts. An example of an Event is a business process. Whenever an Event occurs, it is not the case that all of its temporal parts also occur. For instance, if we consider a business process "Buy a product" at different time instants when it occurs, at each of these time instants only some of its temporal parts are occurring. Finally, we can consider Events as possible transformations from a portion of reality to another, i.e., they may change reality by changing the state of affairs from one (pre-state) situation to a (post-state) situation.

\section{B. Intentional Elements}

UFO has been extended to incorporate intentionality to this basic core $[17,20]$. In this context, UFO distinguishes between Agentive and Non-agentive substantial individuals, termed here Agents and Objects, respectively.

\footnotetext{
${ }^{1}$ Technically, a substantial does not existentially depend on other substantials which are disjoint from it [16].
} 
Agents are substantials capable of bearing special kinds of moments named Intentional Moments. As argued in [33], intentionality should be understood in a much broader context than the notion of "intending something", but as the capacity of some properties of certain individuals to refer to possible situations of reality.

Every intentional moment has a type (e.g., Belief, Desire, Intention) and a propositional content. The latter being an abstract representation of a class of situations referred by that intentional moment. The precise relation between an intentional moment and a situation is the following: a situation in reality can satisfy the propositional content of an intentional moment (i.e., satisfy - in the logical sense - the proposition representing that propositional content).

Whilst a desire expresses a will of an agent towards a state of affairs in reality (e.g., a Desire that Brazil wins the Next World Cup), intentions are desired state of affairs for which the agent commits at pursuing (an intention is an internal commitment) (e.g., the Intention of going to a beach resort for the next summer break) $[11,33]$.

Actions are intentional events, i.e., events with the specific purpose of satisfying (the propositional content of) some Intention of an Agent. (In this sense, an Action can be said to be caused by the Intention.) The propositional content of an intention is termed a Goal. UFO contemplates a relation between Situations and Goals such that a Situation (or possibly a number of Situations) may satisfy a Goal. In other words, since a Goal is a proposition (the propositional content of an Intention), we have that a particular state of affairs can be the truthmaker of that proposition.

\section{Social Elements}

Communicative Acts (special kinds of Actions) can be used to create Social Moments. In this view, language not only represents reality but also creates a part of reality [33]. Thus, social moments are types of intentional moments that are created by the exchange of communicative acts and the consequences of these exchanges (e.g., goal adoption, delegation [18]). For instance, suppose that John rents a car at a car rental service. When signing a business agreement, John performs a communicative act (a promise). This act creates a Social Commitment towards that organization: a commitment to return the car in a certain state, etc (the propositional content). Moreover, it also creates a Social Claim of that organization towards John with respect to that particular propositional content. Commitments/Claims always form a pair that refers to a unique propositional content.

A Social Relator is an example of a relator composed of two or more pairs of associated commitments/claims (social moments). Finally, a commitment (internal or social) is fulfilled by an agent $\mathrm{A}$ if this agent performs an action $\mathrm{x}$ such that the post-state of that action is a situation that satisfies that commitment.

Communicative Acts can also be used to distinguish between Physical Agents (e.g., a person, a dog) and Social Agents (e.g., an organization, a society). Social Agents are created by communicative acts. In an analogous manner, objects can also be categorized as Physical Objects (e.g., cars, rocks and threes) or Social Objects (e.g., a currency, a language, the Brazilian constitution). Social Agents are composed by a number of other agents, which can

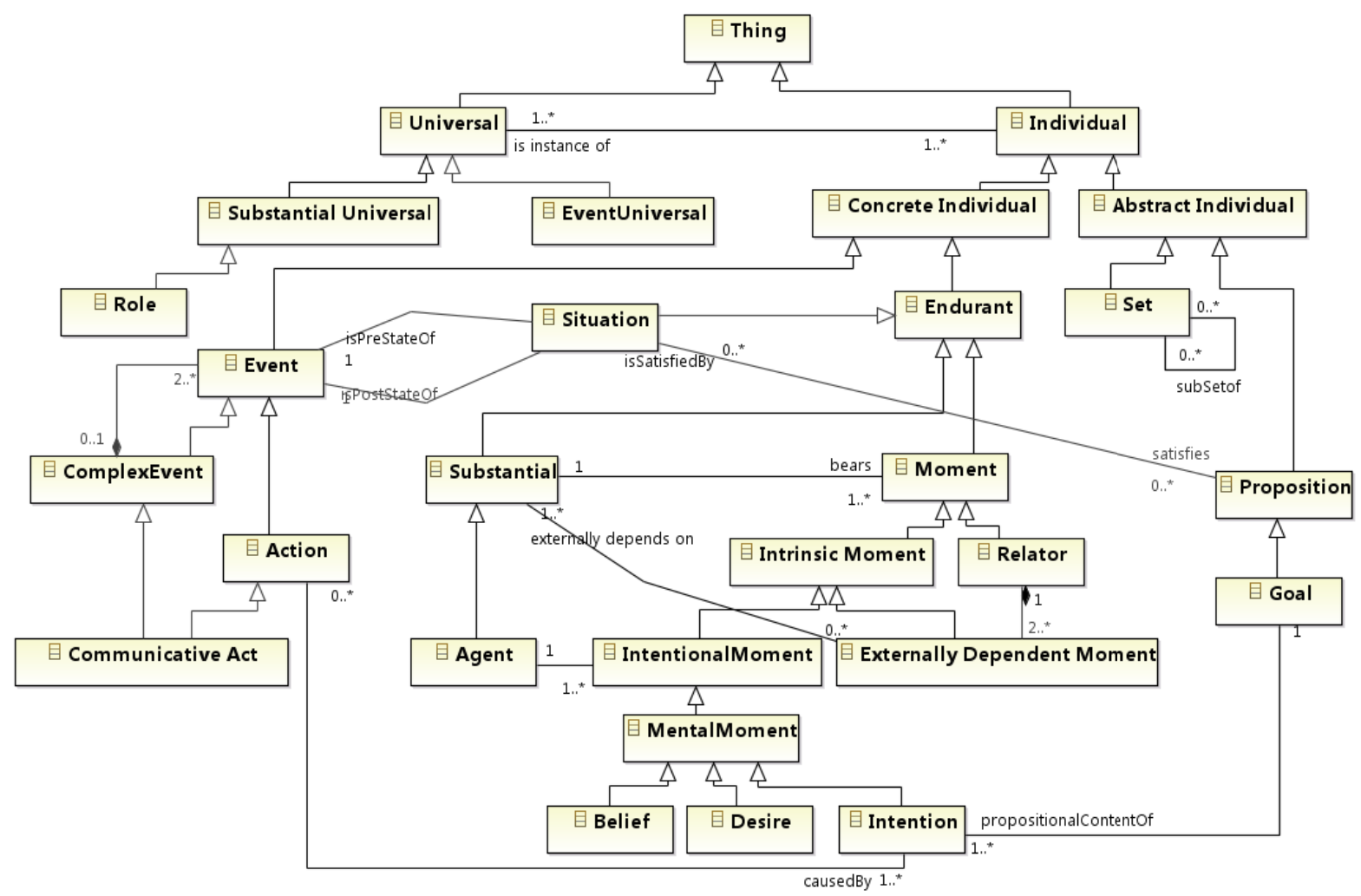

Figure 2 - UFO Fragment (adapted from [16] and [17]) 


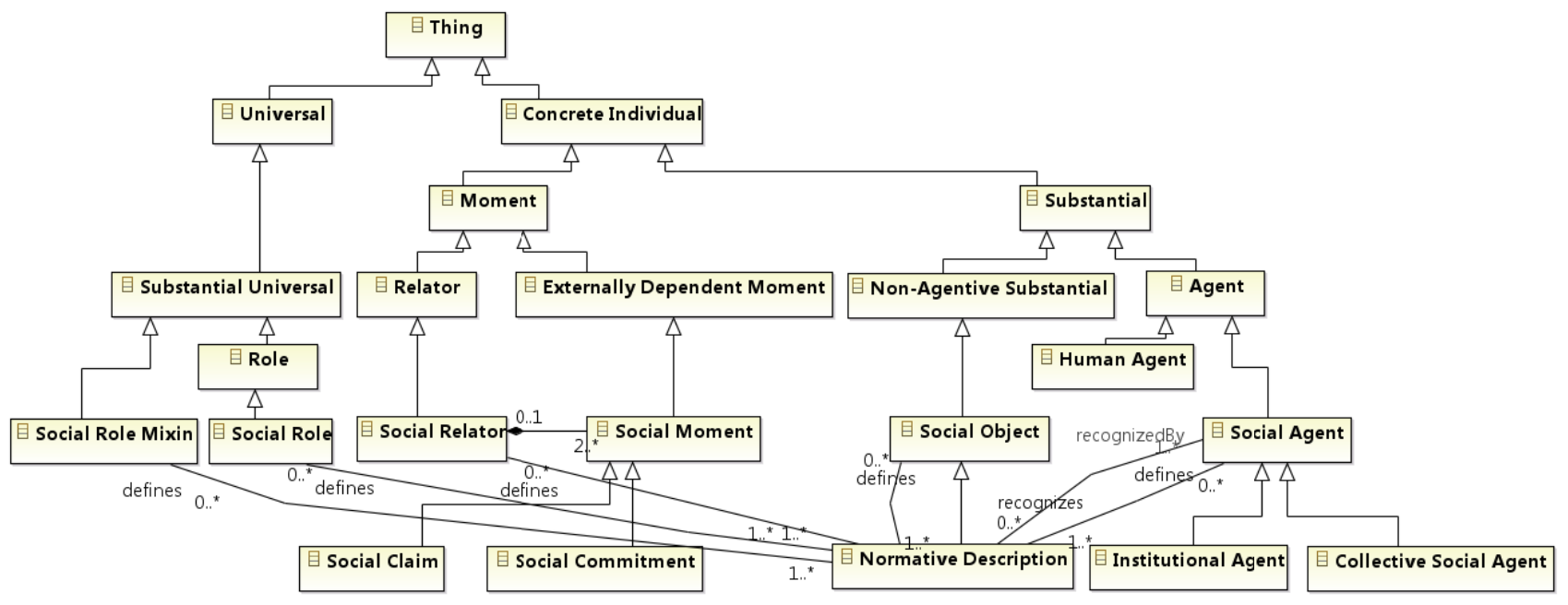

Figure 3 - UFO Fragment (adapted from [16] and [17])

themselves be Physical Agents, or other Social Agents. When Social Agents are integral wholes formed by multiple agents playing different roles they are further classified as Institutional Agents. An Institutional Agent exemplifies what is named a Functional Complex, i.e., a mereologically complex entity whose parts play different roles with respect to the whole. By instantiating each of these roles defined in the characterization of that Functional Complex Universal, each part contributes in a different way to the integral behaviour of the whole.

Communicative Acts can result in social objects called Normative Descriptions. The characteristics of descriptions have been discussed in [28], including (among others) that: "descriptions are created by (communities of) intentional agents at the time of their first encoding in an expression of a 'public' (formal or informal) language"; "descriptions are usually accepted (adopted) by (communities of) intentional agents" and "acceptation can change in time." In the case of a social functional complex such as an Institutional Agent, the characterization of the universal instantiated by that agent is made via a Normative Description [4], which is said to define the institutional agent (including its Social Roles (e.g., president, manager, sales representative), Social Agent Universals (e.g., a political party, an education institution), Social Agents (e.g., the Brazilian Labour Party), Social Object Universals and other Social Objects (e.g., a piece of legislation, a currency) or other Normative Descriptions.

\section{ANALYSIS OF THE ENTERPRISE LANGUAGE}

\section{A. Preliminary Analysis}

Before we attempt an analysis of the objective concept, we must consider the community concept as a key structuring element for enterprise specifications.

A community is "a configuration of enterprise objects that describes a collection of entities (e.g. human beings, information processing systems, resources of various kinds and collections of these) that is formed to meet an objective." A configuration (of objects) is defined in the RM-ODP foundations (Part 2) as "a collection of objects able to interact at interfaces." Thus, a community is a collection of enterprise objectives that are able to interact. The definition seems to suggest some sort of whole-part relation between communities and enterprise objects. Since the enterprise language defines that "the assignment of actions to the enterprise objects that comprise a community is defined in terms of roles", we can consider the relation between community and enterprise objects to be that of type componentOf [16] or Component/Functional Complex [39].

Linington, Milosevic and Raymond clarified the intention behind the community concept in [27]: "The ODP enterprise concept of community is introduced to model how a group of enterprise objects achieve their individual objectives through commonly agreed patterns of interaction. This agreement is institutionalised through the contract. If an object wishes to participate in a community, it is obliged to comply with policies set out in the contract of that particular community."

This suggests that the "unifying condition" [36] among the constituent parts in a community is the "wish" to participate in a community and an agreement to commit to the terms in the community contract. The objects that are part of the community are thus the objects that have entered in this contract. The contract is a Normative Description that defines the Social Roles ${ }^{2}$ and the Social Moment Universals in the scope of the community. Objects that are part of the community bear Social Moments that instantiate the universals defined in this Normative Description. In other words, the objects instantiate the Social Roles defined in the community. This forms a unifying condition so that we can consider a community to be an integral whole instead of a purely formal (mereological) sum (in the senses put forward in [36]) or a system instead of a mere aggregate (in the senses put forward in [8]).

The language used in the text seems to grant enterprise objects an intentional aspect, with the terms "their individual

\footnotetext{
2 There is a technical difference between the notion of role in UFO and the notion of role in RM-ODP. Please refer to [1] for further discussion on this issue.
} 
objectives" and "wish" and the distinction between "physical" and "social" actions. We believe here that the intention of the authors was to emphasize the agentive elements in the community, leaving out information processing systems and resources that have no agentive nature. We will put forward similar emphasis, concluding that agentive enterprise objects in a community form an Institutional Agent. This opens up the community as a potential bearer of intentional moments (such as intentions) and social moments (such as social commitments and claims). Further, since agents that are part of an institutional agent may themselves be institutional agents it enables hierarchical decomposition of enterprise objects as proposed in the enterprise viewpoint.

\section{B. Definition of the Objectives Concept}

The enterprise viewpoint defines the objective (of an $<X>$ ) as a "practical advantage or intended effect, expressed as preferences about future states."

At first observation, this definition seems neutral with respect to the commitments of a community, which seems to imply that the reference model makes no distinction between an objective as the propositional content of a Desire or of an Intention. Nevertheless, objectives are captured in a community as a contract, which establishes how the objective is to be achieved. In this sense, we can say that the community is committed to achieving the objective characterizing the objective as the propositional concept of the community's Intention. Thus we can interpret objectives as Goals in UFO.

UFO contemplates a relation between Situations and Goals such that a Situation (or possibly a number of Situations) may satisfy a Goal. In other words, since a Goal is a Proposition (the propositional content of an Intention), we have that a particular state of affairs can be the truthmaker of that Proposition. We interpret the term state in the RM-ODP definition of objective as Situation. A "preferred" future state is a situation that would satisfy the Goal.

(An alternative would be to ascribe some notion of "utility" to situations in order to be able to compare them. This "utility" could be agent-indexed in order to capture the notion of "perceived utility". In that case, we could discuss "preferences" from the perspective of an agent in terms of the "utility" of possible situations or future states. While we do not pursue this interpretation further in this paper, we by no means intend to rule it out. This interpretation may be particularly interesting to account for the behaviour of agents when facing conflicting objectives and when considering the possibility of violation of norms.)

\section{Objectives, Roles and Objects}

Linington, Milosevic and Raymond have considered the implications of filling a role in a community in the following statement: "In filling a role in a community, the enterprise object is obligated to accept the (direct or delegated) authority of the community over the corresponding participant-roles, and constrain their physical behaviour accordingly."
This statement can be analysed systematically by observing that when an object fills a role in a community there exists a Social Relator between the community and the object (and the object can be said to instantiate some sort of "community member" Social Role). In other words, there is a pair of social commitment/claim between the community and the agent, in which the agent promises to "constrain its physical behaviour" according to the community contract. The propositional content of the commitment/claim pair is defined in the contract (a NormativeDescription).

When an enterprise object commits to performing some sort of behaviour we can regard the relation between the community and the enterprise objects as some sort of plan delegation [17] (or closed delegation [11]).

Since agents are autonomous, their behaviours may violate the contracts leading to undesirable behaviours from the community point of view. Thus, it is up to the agent to internalize a social commitment into an internal commitment (an intention to "constrain its behaviour accordingly") to fulfil its social commitments with the community. Differences between an agent's social commitments and its internal commitments accounts for violations.

\section{Objective Refinement, Roles and Objects}

The reference model defines structuring rules for objectives (Clause 7.7 "Objective rules") such that objectives can be structured and assigned to roles: "An enterprise specification may decompose the objective of a community into sub-objectives. A sub-objective may be assigned to a collection of roles; in that case, the behaviour of the collection of roles is specified to meet the sub-objective and the sub-objective is met by the collection of objects performing the actions of the collection of roles."

This structuring rule implies that (i) objectives can be refined into sub-objectives; and (ii) objectives may be assigned to roles.

With respect to (i), while the reference model defines that "an enterprise specification may decompose the objective of a community into sub-objectives" it does not define the kinds of decompositions that may be considered. Objectives are thus subject to refinement without further qualification in the reference model (differently from behaviours whose refinement is treated explicitly in the reference model.) Our treatment of goals as propositions allows us to account for goal refinement in terms of logical relations between propositions. For example, we can consider two kinds of decompositions here: AND-decomposition and ORdecomposition (reflecting those defined in the goal modelling technique called Tropos [6]). If we have that goals $\mathrm{G}_{1} \ldots \mathrm{G}_{\mathrm{n}}$ AND-decompose goal $\mathrm{G}_{0}$, this relation should be interpreted as: $\left(\mathrm{G}_{0} \leftrightarrow\left(\mathrm{G}_{1} \wedge \mathrm{G}_{2} \wedge \ldots \wedge \mathrm{G}_{\mathrm{n}}\right)\right)$. In an analogous manner, and OR-decomposition $\mathrm{G}_{1} \ldots \mathrm{G}_{\mathrm{n}}$ of goal $\mathrm{G}_{0}$ should be interpreted as: $\left(\mathrm{G}_{0} \leftrightarrow\left(\mathrm{G}_{1} \vee \mathrm{G}_{2} \vee \ldots \vee \mathrm{G}_{\mathrm{n}}\right)\right)$. The recursive combination of AND and OR decompositions can represent a great number of objective refinement relations.

With respect to (ii), we could explain assignment of goals using two sorts of delegation: goal delegation [17] which involves an agent that commits to accomplish a goal on behalf of another agent without committing to particular 
actions and plan delegation which involves specific actions. However, the text for the structuring rule seems to imply that only plan delegation is possible by prescribing that the behaviour of the roles be specified to meeting the subobjective.

\section{E. Nested Communities}

In a hierarchical perspective on community forming, the reference model defines that "a community may be regarded as a composite community-object which may fulfil a role in another community. In this case, the objective of the community of which the community-object is an abstraction is consistent with any sub-objectives assigned to that role in the other community." (We can state that the communityobject A qua member of community B does not have a goal which contradicts (in the logical sense, since goals are propositions) the goals of $\mathrm{B}$ )

Assignment of objectives to roles filled by communityobjects seems to open up the path for goal delegation for community-objects; the delegatum is the sub-objective assigned to the role by "the other community". Since goal delegation seems to be allowed in this case, we argue that the text for the structuring rule in clause 7.7 should be reviewed to avoid proscribing goal delegation for enterprise objects which are not community-objects. We favour that interpretation to grant communities and other enterprise objects uniform treatment.

\section{F. Conformance}

Since agents exhibit autonomous and heterogeneous behaviour, they may be more or less committed with community objectives according to the extent to which their own objectives are compliant with the community's objectives. Again, there is an issue of conformance of the agent's actual behaviour and the behaviour implied by social commitment (what is called "social behaviour" in [27]),

It is worth noting that while [27] uses the term "individual objectives" referring to enterprise objects, this is not explicit in the reference model. We believe the ontological account discussed here corroborates the observations in [27] further emphasizing the importance of the extension of the reference model to account for individual objectives and the possibility of non-conformance.

\section{G. Objectives and Processes}

The structuring rules for objectives (Clause 7.7 "Objective rules") further define that a sub-objective may be assigned to a process ("A collection of steps taking place in a prescribed manner and leading to an objective."). In that case, "the process is specified to meet the sub-objective and the sub-objective is met by the actions of objects performing the process. In this case, the sub-objective defines the state in which the process terminates."

Since an Action (instance of an Action Universal) is an intentional event performed by agents with the purpose of achieving goals, we can interpret a process as the description of a complex action universal - characterizing an intentional event that can be decomposed into other (intentional) events.
Instances of this complex action universal are said to bring about a situation (which is the post-state of the action).

The structuring rule in the reference model seems to define a strict relation between processes and assigned subobjectives: all instances of the described complex action universal must produce a post-situation that satisfies the subobjective ("the sub-objective defines the state in which the process terminates"). The process must therefore only include behaviour that leads directly to the objective. This would characterize what is called "restricted-scope goal" in [9], and would rule out the assignment of "broad-scope goals" [9] to business processes. A broad-scope goal is achieved after one or several executions of a business process. Consider for example, the objective of obtaining $90 \%$ customer approval. This objective can only be considered in the long run and is not applicable in the scope of a single business process execution.

Further, the strict relation between a processes and an assigned sub-objective implies that the process must not include behaviour that may fail to achieve the objective. A process must be seen in this case as a "recipe" to meet the objective and not as "an attempt" to meet the objective.

A less strict relation could be considered to account both for broad-scope goals and for the notion that certain behaviour may increase the likelihood of achieving certain goals, or even that certain behaviour is recommended to achieve certain goals (for example based on previous experience or probabilities). This would enable us to consider "technical norms" [4] and processes for which the outcome is uncertain (involving risk and chance).

\section{H. A Note on the Identity Criteria for Communities}

The text in the reference model uses the term "collection" to define "configuration" and uses the term "configuration" to define "community". The term "collection" is used in its informal sense and no precise definition is given. The term is also used in several definitions of Part 2 and throughout the Enterprise Language standard. As we have discussed, the term suggests some notion of whole-part relation, but may be used to denote different kinds of whole-part relations with different implications to the various definitions. For example, collections may follow an extensional or nonextensional principle of identity [16]. In the case of an extensional principle of identity a change in the composition of the collection (addition or subtraction of members) alters the identity of the collection, rendering a different collection. This is not the case for a non-extensional principle of identity, in which the community identity is preserved in the face of changes to the collection.

If a community is a collection, will a change in the composition of the collection (addition or subtraction of members) render a different community? In this particular case, we can find the answer in Part 2, which states that "the specification of a configuration may be static or may be in terms of the operation of dynamic mechanisms which change the configuration, such as binding and unbinding." The last part of the definition of a configuration suggests that configurations are wholes which can either follow an extensional or non-extensional principle of identity. 
While in this particular case we can find an answer in the definition of configuration regarding the identity criteria for a community, the use of the term "collection" informally conceals the intention behind the term. In other parts of the standard, the term is used to denote whole-part relations for descriptions of Events (e.g., process as a "collection of steps") and the mathematical concept of Set (e.g., "a policy may specify which of some collection of policies is to be applied in certain circumstances.") These concepts have radically different standings in the foundational ontology.

\section{Summary}

Table 1 summarizes the correspondence between the enterprise language concepts and the UFO concepts.

Table 1 - Correspondence between RM-ODP and UFO

\begin{tabular}{|c|c|}
\hline RM-ODP & Ontological concept (from UFO) \\
\hline Community & Institutional Agent \\
\hline Enterprise Object & Agent \\
\hline Enterprise Role & Social Role \\
\hline Process & $\begin{array}{l}\text { A description of an Action Universal } \\
\text { (the instances of which are complex } \\
\text { intentional events) }\end{array}$ \\
\hline Objective & $\begin{array}{l}\text { Goal (propositional content of an Agent's } \\
\text { Intention) that is satisfied by Situations }\end{array}$ \\
\hline Contract & $\begin{array}{l}\text { Description (a kind of Social Object) that } \\
\text { defines Social Relators, Social } \\
\text { Commitments/Claims and Social Roles }\end{array}$ \\
\hline $\begin{array}{l}\text { Objective } \\
\text { refinement }\end{array}$ & $\begin{array}{l}\text { The propositional content of the Goal is the } \\
\text { conjunction/disjunction of the } \\
\text { propositional contents of sub-Goals }\end{array}$ \\
\hline $\begin{array}{c}\text { Assignment of } \\
\text { objective to roles }\end{array}$ & $\begin{array}{l}\text { Plan delegation (and possibly } \\
\text { also Goal delegation) }\end{array}$ \\
\hline $\begin{array}{l}\text { Assignment of } \\
\text { objective to } \\
\text { process }\end{array}$ & $\begin{array}{l}\text { All instances of the action universal } \\
\text { described by the process must produce } \\
\text { a post-situation that satisfies } \\
\text { the goal (objective) }\end{array}$ \\
\hline $\begin{array}{l}\text { Object fills a role } \\
\text { in a community }\end{array}$ & $\begin{array}{l}\text { Object instantiates a Social Role defined in } \\
\text { the community's contract. } \\
\text { There exists a Social Relator between the } \\
\text { community and the object }\end{array}$ \\
\hline
\end{tabular}

\section{CONCLUSIONS}

Methods for enterprise modelling that address strategic concerns aim at describing organizations to facilitate the decision-making process which ultimately determines how an enterprise's objectives are operationalized, possibly with the support of an open distributed system. In this paper, we have reviewed how RM-ODP addresses these strategic concerns by interpreting the RM-ODP concepts in terms of a foundational ontology which incorporates concepts to deal with some aspects of social reality and intentionally. More specifically, we have investigated the concept of objective and its related concepts (community, process, contract, roles).

In line with [27], we propose here interpretations that emphasize the intentionally of communities and enterprise objects. In our view, communities and enterprise objects are potential bearer of intentional moments (such as intentions) and social moments (such as social commitments and claims). Our account of objectives allowed us to discuss the refinement of objectives which is not addressed explicitly in
RM-ODP and also allowed us to make some remarks regarding the structuring rules for assignment of objectives to roles (delegations) and to processes.

We regard the efforts to interpret the enterprise language as an opportunity to validate the usefulness and generality of the foundations we employ here. We have been able to identify the need for further investigation to strengthen the foundations with respect to accountability and policy concepts. The extension of the foundation and an interpretation of the accountability and policy concepts will be the subject of our future work. (Please note that the agent concept in RM-ODP is included in the accountability concepts and does not correspond directly to the notion of UFO Agent.)

Further, in [16] the core of the UFO ontology is defined formally in order to allow for unambiguous interpretation of the intended semantics for concepts. Nevertheless, the social aspects introduced in [17] and used here still require full formal treatment. This is a natural extension of the formalization in [16] and also a topic for further work.

Finally, although the reference model defines rich relations between Objectives and its related concepts (Community, Contract, Roles, Behaviours, Processes or SubObjectives), the UML profile for ODP does not allow a modeller to put this conceptualization at work in an enterprise model, restricting itself to a simple relation between objectives and its sub-objectives (modelled as composition) and the assignment of an objective to a community. Further investigation into extensions of the profile (or combinations of the profile with goal modelling languages) seems necessary to capture the complexity of the strategic concern in enterprise settings.

\section{ACKNOWLEDGEMENTS}

This research is funded by the Brazilian Research Funding Agencies FAPES (grant number 45444080/09) and CNPq (grants number 481906/2009-6 and 309059/2008-9).

\section{REFERENCES}

1. J.P.A. Almeida, and G. Guizzardi, "On the Foundation for Roles in RM-ODP: Contributions from Conceptual Modelling," Proceedings WODPEC 2007, pp. 205-215, 2007 doi: 10.1109/EDOCW.2007.21

2. J.P. Almeida, G. Guizzardi, and P.S. Santos Jr, "Applying and extending a semantic foundation for role-related concepts in enterprise modelling," Enterprise Information Systems, vol. 3, no. 3, 2009, pp. 253 - 277. doi: 10.1080/17517570903046292

3. B. Andersson, et al. "Enterprise Sustainability through the Alignment of Goal Models and Business Models". Proceedings of 3rd International Workshop on Business/IT-Alignment and Interoperability (BUSITAL'08) CEUR Workshop Proceedings. 2008.

4. E. Bottazi, and R. Ferrario, "Preliminaries to a DOLCE Ontology of Organizations," Int'l Journal of Business Process Integration and Management, vol. 4, no. 4, March 2010, pp. 225-238.

5. B.F.B. Braga, J.P.A. Almeida, G. Guizzardi, and A.B. Benevides, "Transforming OntoUML into Alloy: towards conceptual model validation using a lightweight formal method," Innovations in Systems and Software Engineering, vol. 6, Springer, 2010, pp. 55-63. 
6. P. Bresciani, A. Perini, P. Giorgini, F. Giunchiglia, J., Mylopoulos, "Tropos: An Agent-Oriented Software Development Methodology". Autonomous Agents and MultiAgent Systems, 2004.

7. British Ministry of Defence. "MOD Architecture Framework", http://www.mod.uk/DefenceInternet/AboutDefence/WhatWeDo /InformationManagement/MODAF, accessed at 04/05/2010.

8. M. Bunge, 'Ontology I: The Furniture of the World, Treatise on Basic Philosophy, vol. 3, D. Reidel Publishing, New York, 1977.

9. E.C.S. Cardoso. "On the Alignment between Goal Models and Enterprise Models with an Ontological Account". Master Thesis. Federal University of Espírito Santo, Brazil, 2009.

10. E.C.S. Cardoso, P.S. Santos Jr., J.P.A. Almeida, R.S.S. Guizzardi, and G. Guizzardi. "Semantic Integration of Goal and Business Process Modeling". IFIP International Conference on Research and Practical Issues of Enterprise Information Systems (CONFENIS 2010), Natal-RN, Brazil, 2010.

11. C. Castelfranchi, and R. Falcone, "Towards a Theory of Delegation for Agent-Based Systems". Robotics and Autonomous Systems, 24(24), 1998, pp. 141-157.

12. R. Conte, C. Castelfranchi, Cognitive and Social Action, UCL Press, London, 1995.

13. P. Fettke and P. Loos, "Ontological Evaluation of Reference Models Using the Bunge-Wand-Weber Model", Proceedings of the 2003 Americas Conference on Information Systems, August 4-6, Florida, USA, 2003.

14. P. Green, and M. Rosemann, "Ontological Analysis of Business Systems Analysis Techniques: Experiences and Proposals for and Enhanced Methodology", Business Analysis with Ontologies, Idea Publishing Group, pp. 1-27, 2005.

15. P. Green, M. Indulska and M. Rosemann, "A Reference Methodology for Conducting Ontological Analyses". Proceedings of the 23rd International Conference on Conceptual Modelling (ER 2004), pp. 8-12. 2004.

16. G. Guizzardi, "Ontological Foundations for Structural Conceptual Models", 2005,Ph.D. Thesis, CTIT PhD-thesis, University of Twente, The Netherlands.

17. G. Guizzardi, R. Falbo, and R. S. S. Guizzardi, "Grounding Software Domain Ontologies in the Unified Foundational Ontology (UFO): The Case of the ODE Software Process Ontology", XI Iberoamerican Conference on Software Engineering (CIbSE’2008), Recife, Brazil, 2008.

18. R. Guizzardi, G. Guizzardi, A. Perini, J. Mylopoulos, "Towards an Ontological Account of Agent Oriented Goals", Software Engineering for Multi-Agent Systems, vol. 5, Springer-Verlag, 2007.

19. G. Guizzardi, L. Pires and M. van Sinderen, "An OntologyBased Approach for Evaluating the Domain Appropriateness and Comprehensibility Appropriateness of Modeling Languages". ACM/IEEE 8th International Conference on Model Driven Engineering Languages and Systems. Lecture Notes in Computer Science vol. 3713, Springer, 2005.

20. G. Guizzardi, and G. Wagner, "On A Unified Foundational Ontology and some Applications of it in Business Modeling". Open INTEROP Workshop on Enterprise Modelling and Ontologies for Interoperability (at CAiSE 2004), 2004.

21. R.S.S. Guizzardi, G. Guizzardi, "Ontology-Based Transformation Framework from Tropos to AORML", Social Modeling for Requirements Engineering, Cooperative Information Systems Series, MIT Press, Boston, 2010.

22. P. Halleux, L. Mathieu, and B. Andersson. "A Method to Support the Alignment of Business Models and Goal Models". Proceedings of 3rd International Workshop on Business/ITAlignment and Interoperability (BUSITAL'08 at CAiSE 2008) CEUR Workshop Proceedings, Vol. 336, 2008, pp. 120-134.
23. ISO - International Organization for Standardization "Information Technology - Open Distributed Processing - Part 2: Foundations, ISO/IEC 10746-2, ITU-T Recommendation X.902, 1995.

24. ISO - International Organization for Standardization. "Information Technology - Open Distributed Processing Reference Model - Enterprise Language ISO/IEC 15414 | ITUT Recommendation X.911, 2004.

25. ISO - International Organization for Standardization "Information technology - Open distributed processing - Use of UML for ODP”. ISO/IEC 19793:2008.

26. M. Lankhorst. Enterprise Architecture at Work - Modelling, Communication, and Analysis, Springer, 2005.

27. P. Linington, Z. Milosevic, K. Raymond, "Policies in communities: extending the ODP enterprise viewpoint," Proceedings 2nd Int'l Enterprise Distributed Object Computing Workshop, (EDOC '98), 1998, pp.14-24.

28. C. Masolo, L. Vieu, E. Bottazzi, C. Catenacci, R. Ferrario, A Gangemi, N. Guarino, "Social Roles and Their Descriptions", Principles of Knowledge Representation and Reasoning, Proceedings of the Ninth International Conference KR 2004, , AAAI Press, 2004, pp. 267-277.

29. Object Management Group (OMG), "Business Motivation Model (BMM)", http://www.omg.org/spec/BMM/1.0, accessed at $04 / 05 / 2010$

30. D. Quartel, et al. "A Goal-Oriented Requirements Modelling Language for Enterprise". Proceedings of the 13th IEEE International EDOC Conference, EDOC 2009. Auckland, New Zealand, 2009

31. P. Santos, J.P. Almeida and G. Guizzardi, "An Ontology-Based Semantic Foundation for ARIS EPCs", Proceedings of 25th ACM Symposium On Applied Computing (ACM SAC 2010), Sierre, Switzerland. 2010

32. A. Scheer, "ARIS - Business Process Modelling", Springer, 2000.

33. J. Searle, Mind, Language and Society, Basic Books, 2000

34. G. Shanks, E. Tansley and R. Weber, "Using Ontology to Validate Conceptual Models". Communications of the ACM 46. pp. 85-89. 2003

35. A. Sharp and P. McDermott. "Workflow Modelling Tools for Process Improvement and Application Development". Artech House, 2001

36. P.M. Simons, Parts. An Essay in Ontology, Oxford: Clarendon Press, 1987.

37. J. F. Sowa and J. Zachman. "Extending and formalizing the framework for information systems architecture". IBM Systems Journal. vol. 31, 3, 1992

38. USA Department of Defense. "DoD Architecture Framework version 1.5 Volume I: Definitions and Guidelines". http://www.defenselink.mil/cio-nii, accessed at 04/05/2010.

39. M.E. Winston, R. Chaffin, and D. Herrman, "A taxonomy of part-whole relations", Cognitive Science 11 (1987), pp.417444.

40. E. Yu, Modelling Strategic Relationships for Process Reengineering, PhD. Thesis. Department of Computer Science, University of Toronto, 1995.

41. E. Yu, M. Strohmaier, and X. Deng. "Exploring Intentional Modelling and Analysis for Enterprise Architecture". Proceedings of the EDOC 2006 Conf. Workshop on Trends in Enterprise Architecture Research, TEAR. 2006. 